\section{Assessing adolescent personality pathology}

\author{
DREW WESTEN, LISSA DUTRA and JONATHAN SHEDLER
}

\section{Background Personality pathology constitutes a major form of psychopathology in adolescents.}

\begin{abstract}
Aims To examine the reliability and validity of a $\mathrm{Q}$-sort instrument for assessing adolescent personality pathology designed for clinically experienced informants.
\end{abstract}

Method A sample of 294 randomly selected psychiatrists and psychologists each provided data on a current patient, aged 14-18 years. Clinicians completed several measures, including the ShedlerWesten Assessment Procedure for Adolescents (SWAP-200-A).

Results Factor analysis identified II dimensions of adolescent personality: psychopathology/malignant narcissism, dysphoria/inhibition, psychological health, histrionic sexualisation, schizotypy, sexual conflict, emotional dysregulation, anxious obsessionality, peer rejection, delinquent behaviour and attentional dysregulation.

These correlated in predicted ways with a range of criterion variables, including measures of adaptive functioning, Axis II pathology, the Five Factor Model and the Child Behavior Checklist.

\section{Conclusions The SWAP-200-A} shows promise as an instrument for assessing personality pathology in adolescents. Trait dimensions such as delinquent behaviour and emotional dysregulation may prove useful additions to a classification of personality.

Declaration of interest None. Funding detailed in Acknowledgements.
A growing body of research suggests that personality pathology constitutes a major form of psychopathology in adolescents, as it does in adults (Ludolph et al, 1990; Johnson et al, 1999; Levy et al, 1999; Westen \& Chang, 2000). At present there are no widely accepted assessment procedures or diagnoses designed specifically for adolescent personality pathology. Whereas previous research on personality disorder features in adolescents, including our own earlier study (Westen et al, 2003), has focused on types (Axis II categories) or prototypes (personality constellations assessed dimensionally, such as the extent to which the patient matches a prototype of histrionic personality disorder), this study focuses on traits: more specific, less molar constructs such as negative affect or emotional dysregulation. Whether personality pathology in adolescents (or adults) is best considered in terms of traits, types or prototypes, or some combination thereof, is an important question. Our aim is to identify the structure of pathological personality traits in a sample of adolescents described by their treating clinicians using a personality pathology instrument designed for adolescents, and to provide initial data on the validity of these constructs and their measurement.

\section{METHOD}

\section{Sample and procedure}

The study method has been described in detail elsewhere (Westen et al, 2003). Participants were randomly selected $\mathrm{PhD}$ and MD clinicians, who provided quantified data on a randomly selected adolescent patient (operationalised as 'the last patient you saw last week before completing this form who meets study criteria') currently in treatment for 'enduring maladaptive patterns of thought, feeling, motivation, or behaviour - that is, personality'. We used this relatively generic definition of personality pathology to avoid limiting our study to patients meeting diagnostic criteria for personality disorder. We ascertained a sample stratified by gender and age (14-18 years) and asked clinicians to complete a packet of questionnaires for a modest honorarium (US\$25).

\section{Measures}

SWAP-200-A

The 200-item Shedler-Westen Assessment Procedure for Adolescents (SWAP-200-A) is a Q-sort instrument for assessing adolescent personality pathology designed for use by skilled clinical observers based on either longitudinal knowledge of the patient over the course of treatment or a systematic clinical interview of the patient and parents. A Q-sort is a set of statements that provides a 'standard vocabulary' for clinicians to use to describe their clinical observations. To describe a patient, the clinician sorts statements into categories based on their applicability to the patient, from those that are irrelevant or not descriptive to those that are highly descriptive. In this study clinicians used a semi-constrained rating scale version of the instrument (see Westen et al, 2003).

The SWAP-200-A was adapted from the SWAP-200 for adults; both measures have shown initial evidence of reliability and validity (Westen \& Shedler, 1999a,b, 2000; Westen \& Muderrisoglu, 2003). Preliminary research has shown high correlations between SWAP-200 descriptions made by treating clinicians and independent interviewers and between independent observers reviewing recorded interviews (Westen \& Muderrisoglu, 2003). The SWAP-200-A correlates with a range of variables such as attachment status, and history of suicide attempts, psychiatric hospitalisations, arrests, and family and developmental history variables (NakashEisikovits et al, 2003; Westen et al, 2003).

The items reflect constructs from a mixture of sources: Axis II criteria for DSM-III through DSM-IV; selected Axis I criteria associated with personality disturbance (e.g. depression and anxiety); clinical literature and research on personality disorders, normal personality traits and psychological health; a model of functional diagnosis (Westen, 1998); research on child and adolescent personality and psychopathology; videotaped clinical interviews; and feedback from over a thousand experienced clinicians. To develop the adolescent 
version of the instrument, we deleted, revised and added items as appropriate based on the adolescent literature, the authors' prior research and experience with adolescent personality pathology, and consultation with senior clinicians in adolescent psychiatry who used the instrument to describe patients and then provided feedback on items that were ambiguous, necessary for describing their patient but missing from the item set, and so on.

Two features of the SWAP-200-A are of particular relevance to assessment of adolescent personality pathology. First, the instrument is intended for use by clinically experienced observers, based on either all available data over the course of their work with a patient or a systematic clinical interview with the patient and parents, the Clinical Diagnostic Interview for Adolescents (CDI-A; further details available from the author upon request). The SWAP-200-A does not presume that patients, particularly adolescents, can self-report their maladaptive personality traits. Rather, it presumes that a skilled clinical interviewer can listen to patients' narratives, observe their interactions with their parents and the interviewer, and integrate information across informants to make judgements about adolescents' characteristic ways of thinking, feeling, regulating affect and so forth. Second, the instrument can be used to assess Axis II diagnoses in adolescents, by correlating patients' 200 -item profiles with diagnostic prototypes of each personality disorder derived from a normative adult sample (Westen \& Shedler, 1999a). Alternatively, it can be used in taxonomic work, as in this study, to develop nonredundant (i.e. relatively non-comorbid) diagnostic categories or dimensions.

\section{Clinical Data Form}

Following basic demographic and diagnostic questions, the Clinical Data Form (CDF) (see Westen \& Shedler, 1999a) asks clinicians to rate the patient's adaptive functioning, including school functioning (1 severe conduct problems/suspensions, 7 working to potential); peer functioning (1 very poor, 7 very good); history of suicide attempts, arrests and hospitalisations; and social support (number of people in whom the patient feels comfortable confiding). Research has demonstrated that clinician ratings of adaptive functioning variables, including the variables assessed by the $\mathrm{CDF}$, show strong correlations with the same variables obtained by interview (see Westen \& Weinberger, 2004). The CDF also measures family and developmental history variables; however, because we do not analyse those data here, we shall not describe them further.

\section{Axis II pathology}

To maximise reliability of measurement, we assessed Axis II pathology as defined by DSM-IV (American Psychiatric Association, 1994) in more than one way. First, we listed the Axis II disorders and asked clinicians to rate the extent to which the patient met criteria for each disorder on a sevenpoint rating scale. Second, we provided clinicians with a checklist of all Axis II criteria, randomly ordered, and asked them to rate each criterion as present or absent, as in DSM-IV, and then to rate the extent to which each item applied using a sevenpoint scale. These checklist data generated two additional dimensional measures of Axis II pathology (number of diagnostic criteria met for each personality disorder and the mean of the ratings 1-7 for each criterion for each diagnosis), as well as categorical diagnoses derived by summing the number of criteria present and applying DSM-IV thresholds. To maximise reliability, we created a composite measure of personality pathology by transforming the three sets of dimensional scores (global ratings, number of Axis II criteria met and summed seven-point ratings across criteria for each disorder) into $\mathrm{Z}$ scores, which we then averaged to form composite personality disorder ratings.

\section{Five Factor Model adjective checklist}

The Five Factor Model (FFM; McCrae \& Costa, 1997) is a model of personality derived by factor analysis. It isolates five general personality traits: neuroticism, extroversion, openness to experience, agreeableness and conscientiousness. According to the most widespread version of the model, embodied in the NEO Personality Inventory Revised (NEO-PI-R; McCrae \& Costa, 1997), each factor includes six sub-factors or 'facets'. For this study we developed a brief clinician-report FFM adjective checklist, consisting of 35 items rated on a seven-point scale, one for each of the NEO-PI-R factors and one for each of the six facets. Coefficient alphas for the five NEO-PI-R factors were largely acceptable, ranging from 0.64 to 0.92 , with both mean and median greater than 0.80 .

\section{Child Behavior Checklist}

The Child Behavior Checklist (CBCL; Achenbach, 1991) is a widely used questionnaire designed to assess the behavioural problems and social competencies of children aged 4-18 years; it includes 11 problem scales. The CBCL also yields two broadband, higher-order psychopathology scales, 'internalising' and 'externalising'. We asked clinicians to complete the parent-report version of the CBCL, which they were able to do without difficulty. Clinician-reported data on the CBCL show similar psychometric properties to parentreported data, including high internal consistency for the problem scale scores (median coefficient $\alpha>0.80$ ), virtually identical factor structure and predictable correlates suggesting convergent and discriminant validity (Dutra et al, 2004).

\section{Statistical analyses}

We analysed the data as follows. First, we subjected the SWAP-200-A items to exploratory factor analysis (because of the absence of prior research on the factor structure of the instrument). As a preliminary test of the validity of the factors, we then performed a series of analyses. (For simplicity of presentation, in the tables that follow, we indicate criterion variables predicted a priori to be most strongly associated with each factor in bold. To minimise overinterpretation of findings, we focus only on findings that are relevant to our hypotheses, form a coherent pattern, or were not predicted but were significant at $P<0.01$.) In a first set of analyses, we correlated patients' factor-based scores with dimensional measures of personality pathology, to locate them within a nomological net (Cronbach \& Meehl, 1955) provided by the more familiar DSM-IV Axis II diagnoses. We then examined their relation to personality as measured by the FFM adjective checklist and the problem scales of the CBCL. Finally, we assessed the relation between SWAP-200-A factors and adaptive functioning variables selected a priori as likely to be associated with different forms of personality pathology, including ratings of school performance and quality of peer relationships; number of close friends or confidantes; and history of psychiatric hospitalisations, suicide attempts and arrests. 


\section{RESULTS}

\section{Sample characteristics}

The sample consisted of 294 patients. Clinician respondents $(61.4 \%$ psychiatrists, $50.2 \%$ male) were on average highly experienced (mean years of experience post training 13.4, s.d.=9.4). Clinicians varied in theoretical orientations, and most worked in more than one setting. Clinicians tended to know the patients well: the median length of treatment prior to completing the questionnaire was 20 sessions. Patients were evenly distributed by gender $(52.9 \%$ female) and age. The majority (84.9\%) were White, with most of the remaining patients Black or Hispanic. Clinicians rated the patients as $7.5 \%$ poor, $20.9 \%$ working class, $50.7 \%$ middle class and $20.9 \%$ upper class. The most prevalent Axis I diagnoses included major depressive disorder (25.3\%), dysthymic disorder (24.3\%), attention-deficit hyperactivity disorder $(16.1 \%)$, oppositional defiant disorder $(9.0 \%)$ and conduct disorder $(6.1 \%)$.

\section{Factor structure of the SWAP-200-A}

As a first step, we subjected the SWAP-200A items to a principal components analysis, examining the resulting eigenvalues, percentage of variance accounted for by each factor, and scree plot. The scree plot showed a gradual break between 12 and 15 factors. We obtained similar factor structures using 12-15 factors with both varimax (orthogonal) and promax (oblique) solutions and multiple extraction methods. We retained and report here the first 11 of the 12-factor promax (oblique) solution using principal axis factoring. These factors were readily interpretable, reproducible across several estimation procedures and algorithms, and well marked by multiple items. Communalities were all greater than 0.70 , with most between 0.80 and 0.90 , suggesting that the items did in fact include substantial common components. The 11 factors cumulatively accounted for $52 \%$ of the variance. Reliability (coefficient $\alpha$ ) was above 0.80 for all factors except factor $10(\alpha=0.72)$, with a median of 0.86 . Table 1 lists the items that loaded most highly on each of the 11 factors.

The first factor, psychopathy/malignant narcissism, includes items associated with narcissism, disruptive behaviour disorders, hostility, antisocial personality disorder and psychopathy. The second factor, dysphoria/inhibition, includes depression, anhedonia, shame, guilt and a number of related cognitive and emotional processes. It also includes a tendency to be inhibited in a number of domains, including social, motivational and affective inhibition. The third factor, psychological health, reflects the presence of psychological strengths and inner resources, including the capacity to love, find meaning in life experiences and gain insight into the self. Factor 4, histrionic sexualisation, reflects sexuality typically seen in histrionic personality disorder. Patients scoring high in this dimension tend to be sexually provocative and promiscuous; they also have a tendency to fantasise about ideal love but become involved in emotionally charged, unhealthy romantic relationships. The fifth factor, schizotypy, describes patients with subclinical positive and negative symptoms. Patients scoring high on this dimension tend to have barren representations of themselves and others, impoverished thought more generally and emotional flatness or constriction. They also tend to have odd appearances, mannerisms, reasoning processes and/or perceptual experiences. Factor 6, emotional dysregulation, includes a deficiency in the capacity to modulate and regulate affect, so that in such individuals affect tends to spiral out of control, change rapidly, be expressed in intense and unmodified form, and overwhelm reasoning. This emotional dysregulation may lead to selfdestructive attempts to regulate affects, such as suicidality and parasuicidality, self-injury and/or bingeing and purging. The construct of emotional dysregulation is central to contemporary clinical thought, especially with respect to borderline personality disorder (see Westen, 1991; Linehan, 1993; Westen et al, 1997). Of note is its statistical independence from our two negative affect factors tapping dysphoria and anxiety. Factor 7, anxious obsessionality, reflects highly anxious individuals who may experience obsessions, compulsions, phobias and/or panic attacks; these patients tend to develop somatic problems in response to stress. Factor 8 , delinquent behaviour, reflects a tendency to engage in criminal behaviour, misuse drugs and alcohol, run away from home, seek out thrills and adventure and surround oneself with delinquent peers. Factor 9, sexual conflict, is descriptive of patients who are confused about their sexual orientation and appear to be struggling with counternormative sexual desires and mannerisms.
Factor 10, attentional dysregulation, describes a constellation of personality processes associated with attentional deficits, including low tolerance of frustration and irresponsibility. Factor 11, peer rejection, describes adolescents who have poor social skills and tend to be neglected, avoided or bullied by their peers. Adolescents with high scores on this factor tend to lack close friendships and relationships.

\section{Assessing validity}

The factors that emerged are clinically and theoretically coherent, and most resemble factors that emerged from factor analysis of the SWAP-200 Q-sort for adults (Shedler \& Westen, 2004). To assess the validity of these factors (their association with external criteria), we first examined their relation to dimensional personality disorder diagnoses. As can be seen from Table 2, the SWAP-200-A factors tended to be associated with theoretically relevant variables in predictable ways (predicted correlations are in bold). For example, schizotypy and peer rejection were both strongly associated with personality disorders involving social isolation and peculiarity. The emotionally dysregulated and histrionic sexualisation factors were both associated with borderline, histrionic and dependent personality disorders - three disorders that tend to demonstrate significant diagnostic overlap in adult samples. Also of note is that the psychological health factor was negatively associated with most of the personality disorders.

Our next set of analyses examined the association between the SWAP-200-A factors and the FFM checklist (Table 3) and the CBCL (Table 4). As predicted, SWAP-200-A factors involving negative emotions (notably dysphoria/inhibition, anxious obsessionality and emotional dysregulation) were strongly associated with neuroticism, whereas factors involving externalising pathology (particularly malignant narcissism) tended to correlate negatively with agreeableness and conscientiousness. Of note was the negative correlation between schizotypy and openness to experience, which makes theoretical sense in light of the concreteness and affective detachment of patients rated high on this factor, but does not accord with predictions of FFM researchers who have tried to account for schizotypal thinking as extreme openness to experience (e.g. Widiger et al, 
Table I Factor structure of the Shedler-Westen Assessment Procedure for Adolescents

Factor

Factor I: Psychopathy/malignant narcissism

Tends to be critical of others

Tends to be angry or hostile (whether consciously or unconsciously)

Tends to hold grudges; may dwell on insults or slights for long periods

Tends to get into power struggles with adults

Appears to gain pleasure or satisfaction by being sadistic, aggressive or bullying (whether consciously or unconsciously)

Tends to react to criticism with feelings of rage or humiliation

Tends to blame others for own failures or shortcomings; tends to believe his/her problems are caused by external factors

Tends to see own unacceptable feelings or impulses in other people instead of in him/herself

Tends to be rebellious or defiant toward authority figures

Manages to elicit in others feelings similar to those he or she is experiencing (e.g. when angry, acts in such a way as to provoke anger in others;

when anxious, acts in such a way as to induce anxiety in others)

Tends to express intense and inappropriate anger, out of proportion to the situation at hand

Tends to be oppositional, contrary or quick to disagree

Tends to be controlling

Takes advantage of others; is out for number one; has minimal investment in moral values

Tends to show reckless disregard for the rights, property or safety of others

Draws pleasure or self-esteem from being, or being seen as, 'bad' or 'tough'

Tends to see certain others as 'all bad', and loses the capacity to perceive any positive qualities the other person may have

Is quick to assume that others wish to harm or take advantage of him/her; tends to perceive malevolent intentions in others' words and actions

Tends to be arrogant, haughty or dismissive

Tends to be competitive with others (whether consciously or unconsciously)

Tends to seek power or influence with peers (whether in beneficial or destructive ways)

Seeks to dominate an important other (e.g. sibling, parent, boyfriend, girlfriend) through violence or intimidation

Tends to feel misunderstood, mistreated or victimised

Tends to break things or become physically assaultive when angry

Has an exaggerated sense of self-importance; tends to boast or brag

Tries to manipulate others' emotions to get what $s /$ he wants

Appears to feel privileged and entitled; expects preferential treatment

Has little empathy; seems unable to understand or respond to others' needs and feelings unless they coincide with his/her own

Tends to elicit extreme reactions or stir up strong feelings in others

Tends to elicit dislike or animosity in others

Appears to experience no remorse for harm or injury caused to others

Tends to be deceitful; tends to lie or mislead

Tends to be unconcerned with the consequences of his/her actions; appears to feel immune or invulnerable

Factor 2: Dysphoria/inhibition

Appears to find little or no pleasure, satisfaction or enjoyment in life's activities

Tends to feel $s /$ he is inadequate, inferior or a failure

Tends to be insufficiently concerned with meeting own needs; appears not to feel entitled to get or ask for things s/he deserves

Tends to feel unhappy, depressed, or despondent

Tends to feel life has no meaning

Tends to be shy or reserved in social situations

Appears inhibited about pursuing goals or successes; aspirations or achievements tend to be below his/her potential

Tends to be inhibited or constricted; has difficulty allowing self to acknowledge or express wishes and impulses

Has difficulty allowing self to experience strong pleasurable emotions (e.g. excitement, joy, pride)

Tends to feel listless, fatigued or lacking in energy

Tends to express aggression in passive and indirect ways (e.g. may make mistakes, procrastinate, forget, become sulky, etc.) 


\begin{tabular}{|c|c|}
\hline Factorl & Loading \\
\hline Tends to feel helpless, powerless or at the mercy of forces outside his/her control & 0.50 \\
\hline Tends to feel like an outcast or outsider; feels as if s/he does not truly belong & 0.47 \\
\hline Tends to feel $s /$ he is not his/her true self with others; tends to feel false or fraudulent & 0.47 \\
\hline Tends to deny or disavow own needs for caring, comfort, closeness, etc., or to consider such needs unacceptable & 0.47 \\
\hline Appears to want to 'punish' self; creates situations that lead to unhappiness, or actively avoids opportunities for pleasure and gratification & 0.46 \\
\hline Tends to feel empty & 0.44 \\
\hline Tends to avoid social situations because of fear of embarrassment or humiliation & 0.44 \\
\hline Tends to be self-critical; sets unrealistically high standards for self and is intolerant of own human defects & 0.43 \\
\hline Is simultaneously needy of, and rejecting toward, others (e.g. craves intimacy and caring, but tends to reject it when offered) & 0.42 \\
\hline Has trouble making decisions; tends to be indecisive or to vacillate when faced with choices & 0.42 \\
\hline Tends to feel guilty & 0.42 \\
\hline Generally finds contentment and happiness in life's activities & 0.75 \\
\hline Is creative; is able to see things or approach problems in novel ways & 0.72 \\
\hline Is able to find meaning and satisfaction in the pursuit of goals and ambitions & 0.72 \\
\hline Is psychologically insightful; is able to understand self and others in subtle and sophisticated ways & 0.72 \\
\hline Is able to form close and lasting friendships characterised by mutual support and sharing of experiences & 0.71 \\
\hline $\begin{array}{l}\text { Is resilient in the face of extreme stress; seems to be able to face loss, trauma or deeply troubling events with appropriate feeling and continue } \\
\text { functioning effectively }\end{array}$ & 0.71 \\
\hline Has the capacity to recognise alternative viewpoints, even in matters that stir up strong feelings & 0.70 \\
\hline $\begin{array}{l}\text { Is capable of hearing information that is emotionally threatening (i.e. that challenges cherished beliefs, perceptions and self-perceptions) and } \\
\text { can use and benefit from it }\end{array}$ & 0.70 \\
\hline Appreciates and responds to humour & 0.70 \\
\hline Is empathic; is sensitive and responsive to other peoples' needs and feelings & 0.65 \\
\hline Is able to assert him/herself effectively and appropriately when necessary & 0.65 \\
\hline Enjoys challenges; takes pleasure in accomplishing things & 0.63 \\
\hline Tends to express affect appropriate in quality and intensity to the situation at hand & 0.62 \\
\hline Tends to be conscientious and responsible & 0.60 \\
\hline Tends to be energetic and outgoing & 0.57 \\
\hline Tends to elicit liking in others & 0.53 \\
\hline Finds meaning in belonging and contributing to a larger community (e.g. volunteer organisations, church, neighbourhood) & 0.51 \\
\hline Is able to find meaning and fulfilment in guiding, mentoring or nurturing others & 0.49 \\
\hline Is articulate; can express self well in words & 0.47 \\
\hline Tends to seek out or create interpersonal relationships in which s/he is in the role of caring for, rescuing or protecting the other & 0.44 \\
\hline Appears comfortable and at ease in social situations & 0.41 \\
\hline \multicolumn{2}{|l|}{ Factor 4: Histrionic sexualisation } \\
\hline Tends to be overly sexually seductive or provocative, whether consciously or unconsciously (e.g. may be inappropriately flirtatious) & 0.88 \\
\hline Tends to have numerous sexual involvements; is promiscuous for a person of his/her age & 0.81 \\
\hline Fantasises about finding ideal, perfect love & 0.78 \\
\hline $\begin{array}{l}\text { Tends to become attached quickly or intensely; develops feelings, expectations, etc. that are not warranted by the history or context of the } \\
\text { relationship }\end{array}$ & 0.75 \\
\hline Tends to choose sexual or romantic partners who seem inappropriate in terms of age, status (e.g. social, economic, intellectual), etc. & 0.75 \\
\hline Tends to use his/her physical attractiveness to an excessive degree to gain attention or notice & 0.75 \\
\hline $\begin{array}{l}\text { Tends to become involved in romantic or sexual 'triangles' (e.g. is most interested in partners who are already attached or sought } \\
\text { by someone else) }\end{array}$ & 0.68 \\
\hline Tends to become attached to, or romantically interested in, people who are emotionally unavailable & 0.64 \\
\hline Tends to be drawn into relationships outside the family in which s/he is emotionally or physically abused & 0.58 \\
\hline Interpersonal relationships tend to be unstable, chaotic and rapidly changing & 0.57 \\
\hline
\end{tabular}




\begin{tabular}{|c|c|}
\hline Factorl & Loading \\
\hline Tends to idealise certain others in unrealistic ways; sees them as 'all good', to the exclusion of commonplace human defects & 0.57 \\
\hline Is preoccupied with sex & 0.56 \\
\hline Tends to be suggestible or easily influenced & 0.53 \\
\hline Appears to fear being alone; may go to great lengths to avoid being alone & 0.51 \\
\hline Tends to fear s/he will be rejected or abandoned by those who are emotionally significant & 0.47 \\
\hline $\begin{array}{l}\text { Tends to identify with admired others to an exaggerated degree; tends to become an admirer or 'disciple' (e.g. may take on the other's } \\
\text { attitudes, beliefs and mannerisms) }\end{array}$ & 0.45 \\
\hline Beliefs and expectations seem clichéd or stereotypical, as if taken from books or films & 0.43 \\
\hline Expresses emotion in exaggerated and theatrical ways & 0.43 \\
\hline Tends to be overly needy or dependent (e.g. requires excessive reassurance or approval, is 'clingy' with friends or parents) & 0.41 \\
\hline Seeks to be the centre of attention & 0.41 \\
\hline \multicolumn{2}{|l|}{ Factor 5: Schizotypy } \\
\hline Is not verbally articulate; has limited ability to express self in words & 0.70 \\
\hline $\begin{array}{l}\text { Appearance or manner seems odd or peculiar (e.g. grooming, hygiene, posture, eye contact, speech rhythms, etc. seem somehow strange } \\
\text { or 'off') }\end{array}$ & 0.64 \\
\hline $\begin{array}{l}\text { Reasoning processes or perceptual experiences seem odd and idiosyncratic (e.g. may make seemingly arbitrary inferences; may see hidden } \\
\text { messages or special meanings in ordinary events) }\end{array}$ & 0.61 \\
\hline Speech tends to be circumstantial, vague, rambling, digressive & 0.60 \\
\hline Tends to elicit boredom in others (e.g. may talk incessantly, without feeling or about inconsequential matters) & 0.59 \\
\hline Tends to think in concrete terms and interpret things in overly literal ways; has limited ability to appreciate metaphor, analogy or nuance & 0.55 \\
\hline $\begin{array}{l}\text { Seems to know less about the ways of the world than might be expected, given his/her intelligence, background and age; appears naïve } \\
\text { or innocent }\end{array}$ & 0.52 \\
\hline Appears to experience the past as a series of disjointed or disconnected events; has difficulty giving a coherent account of his/her life or actions & 0.51 \\
\hline Appears to have little need for human company or contact; is genuinely indifferent to the presence of others & 0.50 \\
\hline Appears to have a limited or constricted range of emotions & 0.49 \\
\hline Struggles with genuine wishes to kill him/herself & 0.69 \\
\hline Tends to make repeated suicidal threats or gestures, either as a 'cry for help' or as an effort to manipulate others & 0.65 \\
\hline Tends to engage in self-mutilating behaviour (e.g. cutting, burning) & 0.64 \\
\hline Tends to be preoccupied with death and dying & 0.51 \\
\hline Has uncontrolled eating binges followed by 'purges' (e.g. makes self vomit, abuses laxatives, fasts); has bulimic episodes & 0.48 \\
\hline Tends to enter altered, dissociated state of consciousness when distressed (e.g. the self or the world feels strange, unfamiliar or unreal) & 0.45 \\
\hline $\begin{array}{l}\text { Repeatedly re-experiences or re-lives a past traumatic event (e.g. has intrusive memories or recurring dreams of the event; is startled } \\
\text { or terrified by present events that resemble or symbolise the past event) }\end{array}$ & 0.41 \\
\hline Tends to be preoccupied with food, diet or eating & 0.41 \\
\hline Emotions tend to spiral out of control, leading to extremes of anxiety, sadness, rage or excitement & 0.40 \\
\hline Emotions tend to change rapidly and unpredictably & 0.40 \\
\hline \multicolumn{2}{|l|}{ Factor 7: Anxious obsessionality } \\
\hline $\begin{array}{l}\text { Tends to be preoccupied with concerns about dirt, cleanliness and contamination (e.g. drinking from another person's glass, sitting on public } \\
\text { toilet seats) }\end{array}$ & 0.68 \\
\hline $\begin{array}{l}\text { Has panic attacks lasting from a few minutes to a few hours, accompanied by strong physiological responses (e.g. racing heart, shortness } \\
\text { of breath, feelings of choking, nausea, dizziness) }\end{array}$ & 0.68 \\
\hline Has a specific phobia (e.g. of snakes, spiders, dogs, aeroplanes, lifts) & 0.67 \\
\hline Has unfounded fears of contracting medical illness; tends to interpret normal aches and pains as symptomatic illness; is hypochondriacal & 0.66 \\
\hline Is troubled by recurrent obsessional thoughts that $\mathbf{s} /$ he experiences as senseless and intrusive & 0.52 \\
\hline Tends to adhere rigidly to daily routines and become anxious or uncomfortable when they are altered & 0.52 \\
\hline Tends to develop somatic symptoms in response to stress or conflict (e.g. headache, backache, abdominal pain, asthma) & 0.48 \\
\hline Tends to be anxious & 0.43 \\
\hline Tends to be overly concerned with rules, procedures, order, organisation and schedules & 0.39 \\
\hline
\end{tabular}




\begin{tabular}{|c|c|}
\hline Factor' & Loading \\
\hline \multicolumn{2}{|l|}{ Factor 8: Delinquent behaviour } \\
\hline Tends to misuse illicit drugs & 0.72 \\
\hline Tends to misuse alcohol & 0.69 \\
\hline Tends to engage in unlawful or criminal behaviour & 0.64 \\
\hline Tends to surround him/herself with peers who are delinquent or deeply alienated & 0.52 \\
\hline Tends to seek thrills, novelty and adventure & 0.46 \\
\hline Tends to run away from home & 0.43 \\
\hline \multicolumn{2}{|l|}{ Factor 9: Sexual conflict } \\
\hline Is unsure whether s/he is heterosexual, homosexual or bisexual & 0.63 \\
\hline $\begin{array}{l}\text { Tends to express qualities, mannerisms or attitudes traditionally associated with the opposite gender (e.g. an effeminate boy, or a girl who } \\
\text { disparages anything traditionally feminine) }\end{array}$ & 0.58 \\
\hline Has conscious homosexual interests (moderate placement implies bisexuality; high placement implies exclusive homosexuality) & 0.56 \\
\hline $\begin{array}{l}\text { Shows evidence of unconscious homosexual wishes or interests (e.g. may be excessively homophobic, or may show signs of unacknowledged } \\
\text { attraction to a person of the same gender) }\end{array}$ & 0.40 \\
\hline \multicolumn{2}{|l|}{ Factor 10: Attentional dysregulation } \\
\hline Tends to be unreliable and irresponsible (e.g. may fail to meet school or work obligations) & 0.63 \\
\hline Tends to feel bored & 0.58 \\
\hline Is easily frustrated (e.g. 'gives up' quickly, has trouble accepting appropriate limits) & 0.54 \\
\hline Is inattentive or easily distracted; has trouble concentrating & 0.52 \\
\hline Tends to use his/her psychological or medical problems to avoid school, work or responsibility (whether consciously or unconsciously) & 0.51 \\
\hline Behaviour at school or work is erratic, unpredictable or grossly inappropriate (e.g. is truant or severely disruptive in class) & 0.43 \\
\hline \multicolumn{2}{|l|}{ Factor II: Peer rejection } \\
\hline Tends to be ignored, neglected or avoided by peers & 0.66 \\
\hline Tends to be bullied or teased by peers & 0.62 \\
\hline Lacks social skills; tends to be socially awkward or inappropriate & 0.57 \\
\hline Lacks close friendships and relationships & 0.43 \\
\hline
\end{tabular}

I. Included are items loading above 0.50 (factor I) or 0.40 (factors $2-11$ ). As common in factor-analytic research, we progressively relaxed criteria for inclusion (from 0.50 to 0.40 ) to maximise reliability of the smaller factors. Factor 7 includes an item loading 0.39 because this item was clearly conceptually related to the factor's other items.

2002). With respect to CBCL variables, SWAP-200-A factors with item content suggesting negative emotionality were most highly associated with the internalising score and related sub-scales, whereas factors suggesting externalising pathology correlated most highly with the externalising scale and its component scales.

Finally, we examined the relationship between the SWAP-200-A factors and adaptive functioning variables (Table 5). The data provided additional preliminary support for validity. For example, psychopathy/malignant narcissism correlated with all six variables in the expected directions and also predicted history of trouble with the law. Although most of the SWAP200-A factors predicted poor peer relationships, the correlations were particularly large for schizotypy and peer rejection. The psychological health factor also performed as expected. Of particular interest is the strong pattern of associations between SWAP-200-A factors and variables such as history of suicide attempts, arrests and psychiatric hospitalisation, which are relatively objective and require minimal clinical inference (and hence are not readily attributable to clinician biases).

\section{DISCUSSION}

The SWAP-200-A shows promise as an instrument for assessing personality pathology in adolescents. Its factors are theoretically and clinically coherent, internally consistent, and show convergent and discriminant validity in predicting a range of variables including Axis II diagnosis, FFM scores, CBCL scores and measures of adaptive functioning.

Several aspects of the factor solution are interesting from a conceptual and clinical point of view. The factor structure of the adolescent instrument strongly resembles the factor structure obtained using the adult version of the instrument (Shedler \& Westen, 2004), with a few important exceptions. Whereas the adult instrument yields separate factors for narcissism, psychopathy and hostility, the SWAP-200-A yields a large first factor that blends these constructs, and includes a separate factor that assesses delinquent behaviour. These differences may reflect developmental differences in the expression of the underlying traits, or they may reflect instability of the factor solution reflecting sample size. A distinction between a core psychopathy factor and a delinquent behaviour factor, however, mirrors results of factor analysis of the Psychopathy Checklist Revised, which similarly distinguishes a callous, hostile, externalising personality style from a tendency to become involved in criminal activity (Hare, 1998). Other differences between the adult and adolescent factor structures seem to reflect 
Table 2 Correlations between factor scores and composite personality disorder ratings

\begin{tabular}{|c|c|c|c|c|c|c|c|c|c|c|}
\hline \multirow[t]{3}{*}{ SWAP-200-A factor } & \multicolumn{10}{|c|}{ Composite personality disorder ratings $(n=285)$} \\
\hline & \multicolumn{3}{|c|}{ Cluster A } & \multicolumn{4}{|c|}{ Cluster B } & \multicolumn{3}{|c|}{ Cluster C } \\
\hline & Paranoid & Schizoid & Schizotypal & Antisocial & Borderline & Histrionic & Narcissistic & Avoidant & Dependent & Obsessive \\
\hline I Psychopathy/malignant narcissism & $0.50 * * *$ & $0.33 * * *$ & $0.27^{* * *}$ & $0.70^{1, * *}$ & $0.46 * * *$ & $0.35 * * *$ & $0.75 * * *$ & 0.04 & 0.09 & 0.05 \\
\hline 2 Dysphoria/inhibition & $0.30 * * *$ & $0.41^{* * *}$ & $0.40 * * *$ & -0.09 & $0.33 * * *$ & 0.11 & 0.00 & $0.67 * * *$ & $0.53 * * *$ & $0.30 * * *$ \\
\hline 3 Psychological health & $-0.28 * * *$ & $-0.5 I^{* * *}$ & $-0.35^{* * *}$ & $-0.44 * * *$ & $-0.32^{* * *}$ & $-0.13^{* * *}$ & $-0.3 I^{* * *}$ & $-0.21 * * *$ & -0.15 & 0.01 \\
\hline 4 Histrionic sexualisation & $0.35^{* * *}$ & 0.08 & $0.19 * * *$ & $0.22 * * *$ & $0.63^{* * *}$ & $0.72 * * *$ & $0.36 * * *$ & 0.11 & $0.47^{* * *}$ & 0.09 \\
\hline 5 Schizotypy & $0.4 I^{* * *}$ & $0.63 * * *$ & $0.62 * * *$ & $0.33^{* * *}$ & $0.23^{* * *}$ & $0.22 * * *$ & $0.26 * * *$ & $0.40 * * *$ & $0.33^{* * *}$ & $0.30 * * *$ \\
\hline 6 Emotional dysregulation & $0.24 * * *$ & 0.13 & $0.21 * * *$ & 0.08 & $0.68 * * *$ & $0.4 I^{* * *}$ & $0.15^{* *}$ & $0.18^{* *}$ & $0.36 * * *$ & 0.11 \\
\hline 7 Anxious obsessionality & $0.13^{* *}$ & $0.18^{* *}$ & $0.29 * * *$ & $-0.16^{* *}$ & 0.10 & 0.11 & 0.21 & $0.36 * * *$ & $0.33^{* * *}$ & $0.57 * * *$ \\
\hline 8 Delinquent behaviour & $0.28 * * *$ & 0.11 & 0.04 & $0.69 * * *$ & $0.36 * * *$ & $0.32^{* * *}$ & $0.35^{* * *}$ & -0.08 & 0.02 & -0.12 \\
\hline I0 Attentional dysregulation & $0.33^{* * *}$ & $0.35^{* * *}$ & $0.32 * * *$ & $0.49 * * *$ & $0.39 * * *$ & $0.35^{* * *}$ & $0.36 * * *$ & $0.31 * * *$ & $0.33^{* * *}$ & -0.10 \\
\hline II Peer rejection & $0.37 * * *$ & $0.58 * * *$ & $0.62 * * *$ & $0.15^{* *}$ & $0.19 * *$ & $0.16 * *$ & $0.20 * * *$ & $0.52 * * *$ & $0.28 * * *$ & $0.26 * * *$ \\
\hline
\end{tabular}

I. Criterion variables predicted to be most strongly associated (positively or negatively) with each factor are identified in bold.

SWAP-200-A, Shedler-Westen Assessment Procedure for Adolescents.

$* P<0.05, * * P<0.01$, $* * * P<0.001$.

developmental differences. For example, where the adult instrument yields a schizoid factor and a thought disorder (schizotypy) factor, the adolescent version yields a single schizotypy factor and a separate peer rejection factor. Further, although both versions of the instrument produced a sexual conflict factor, in adolescents the items focus primarily on conflicts regarding sexual orientation, probably reflecting the salience of this issue in adolescents struggling with homosexual feelings, and the lack of knowledge both teenagers and their clinicians are likely to have about other kinds of sexual conflict that might not become expressed until adulthood.

Also notable is the distinction between dysphoria/inhibition (the factor most closely related to negative affectivity or neuroticism), anxious obsessionality and emotional dysregulation, three variables that were only moderately intercorrelated. The distinction between negative affectivity on the one hand and emotional dysregulation on the other has emerged recently in other samples using both factor and
Q-factor analysis with a variety of instruments (e.g. Livesley et al, 1998; Westen \& Shedler, 1999b; Westen et al, 2003). Whereas most factor-analytically derived models of personality and mood distinguish positive and negative affectivity - or (in the FFM) their close cousins, extroversion and neuroticism - the emergence of an independent emotional dysregulation factor in clinical samples with instruments intended for clinical use may be significant, drawing attention to the distinction between stably anxious or dysphoric

Table 3 Correlations between factor scores and Five Factor Model Checklist ratings

\begin{tabular}{|c|c|c|c|c|c|}
\hline \multirow[t]{2}{*}{ SWAP-200-A factor } & \multicolumn{5}{|c|}{ Five Factor Model ratings $(n=284)$} \\
\hline & Neuroticism & Extroversion & Agreeableness & Conscientiousness & Openness \\
\hline I Psychopathy/malignant narcissism & 0.14 & 0.06 & $-0.58^{1, * * *}$ & $-0.49 * * *$ & $-0.16^{* *}$ \\
\hline 2 Dysphoria/inhibition & $0.55^{* * *}$ & $-0.38 * * *$ & 0.03 & -0.04 & 0.00 \\
\hline 3 Psychological health & -0.08 & $0.59 * * *$ & $0.70 * * *$ & $0.66^{* * *}$ & $0.65^{* * *}$ \\
\hline 4 Histrionic sexualisation & $0.30 * * *$ & $0.15^{* *}$ & $0.18^{* *}$ & $-0.25^{* * *}$ & 0.11 \\
\hline 5 Schizotypy & 0.15 & $-0.30 * * *$ & $-0.30 * * *$ & $-0.32^{* * *}$ & $-0.22^{* * *}$ \\
\hline 6 Emotional dysregulation & $0.46 * * *$ & -0.01 & -0.09 & -0.12 & 0.01 \\
\hline 7 Anxious obsessionality & $0.38^{* * *}$ & $-0.15^{* *}$ & 0.14 & $0.20 * * *$ & 0.06 \\
\hline 8 Delinquent behaviour & 0.04 & $0.24 * * *$ & $-0.28 * * *$ & $-0.43 * * *$ & 0.04 \\
\hline 9 Sexual conflict & $0.21 * * *$ & 0.01 & -0.01 & 0.00 & $0.16 * *$ \\
\hline I0 Attentional dysregulation & $0.35^{* * *}$ & -0.10 & $-0.33^{* * *}$ & $-0.56 * * *$ & -0.13 \\
\hline II Peer rejection & $0.23^{* * *}$ & $-0.43 * * *$ & $-0.28 * * *$ & $-0.25^{* * *}$ & -0.13 \\
\hline
\end{tabular}

I. Criterion variables predicted to be most strongly associated (positively or negatively) with each factor are identified in bold. SWAP-200-A, Shedler-Westen Assessment Procedure for Adolescents.

$* P<0.05, * * P<0.01, * * * P<0.001$. 


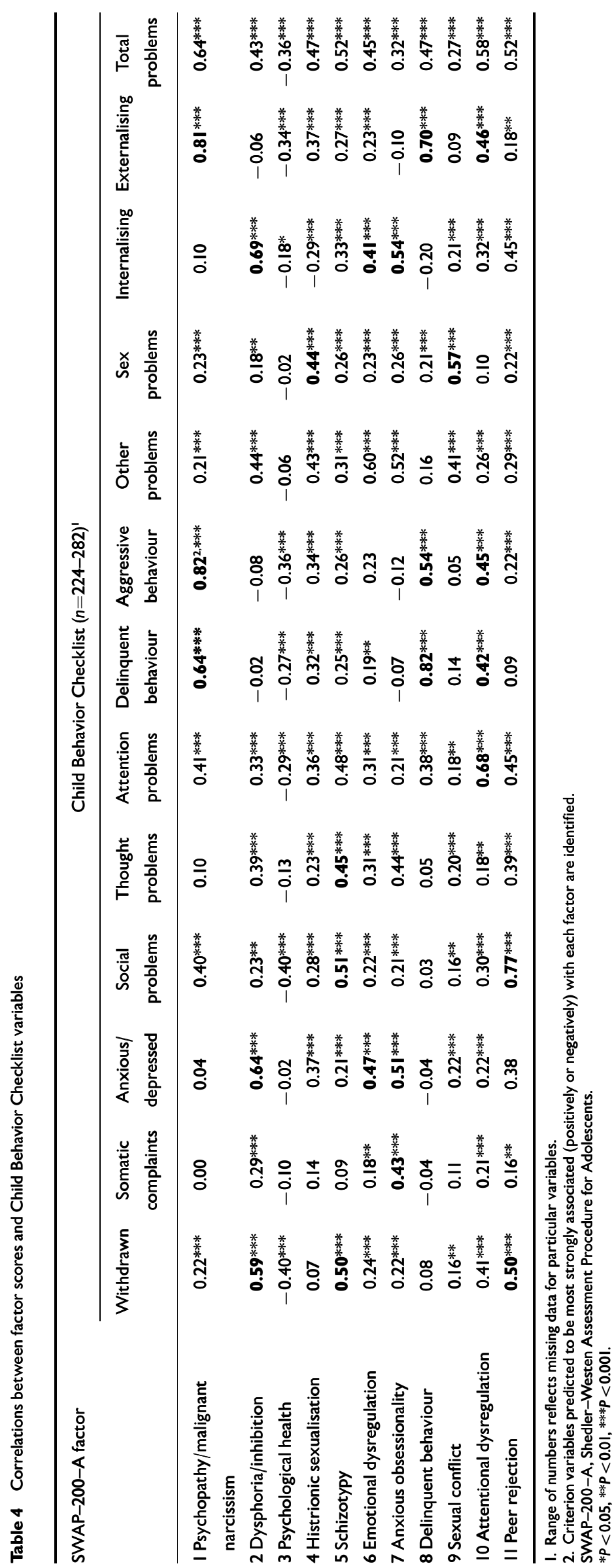




\begin{tabular}{|c|c|c|c|c|c|c|}
\hline \multirow[t]{2}{*}{ SWAP-200-A factor } & \multicolumn{6}{|c|}{ Adaptive functioning variables $(n=242-283)^{\prime}$} \\
\hline & $\begin{array}{c}\text { School } \\
\text { functioning }\end{array}$ & $\begin{array}{l}\text { Quality of peer } \\
\text { relationships }\end{array}$ & $\begin{array}{l}\text { Number of close } \\
\text { relationships }\end{array}$ & $\begin{array}{l}\text { Past psychiatric } \\
\text { hospitalisations }\end{array}$ & $\begin{array}{l}\text { Suicide } \\
\text { history }\end{array}$ & $\begin{array}{l}\text { Arrest } \\
\text { history }\end{array}$ \\
\hline I Psychopathy/malignant narcissism & $-0.42^{2, * * *}$ & $-0.35 * * *$ & $-0.22 * * *$ & $0.20 * *$ & $0.19 * * *$ & $0.25 * * *$ \\
\hline 2 Dysphoria/inhibition & -0.07 & $-0.27 * * *$ & -0.14 & $0.19 * *$ & $0.20 * * *$ & -0.03 \\
\hline 3 Psychological health & $0.51 * * *$ & $0.64^{* * *}$ & $0.42 * * *$ & $-0.34 * * *$ & $-\mathbf{0 . 1 7 * *}$ & $-0.17^{* *}$ \\
\hline 4 Histrionic sexualisation & -0.06 & $-0.20 * * *$ & 0.02 & $0.18^{* *}$ & $0.30 * * *$ & 0.00 \\
\hline 5 Schizotypy & $-0.24 * * *$ & $-0.44 * * *$ & $-0.32 * * *$ & 0.14 & 0.04 & 0.10 \\
\hline 6 Emotional dysregulation & -0.06 & -0.15 & -0.06 & $0.36 * * *$ & $0.42 * * *$ & -0.06 \\
\hline 7 Anxious obsessionality & 0.15 & -0.11 & -0.07 & 0.06 & 0.03 & -0.09 \\
\hline 8 Delinquent behaviour & $-0.40 * * *$ & -0.04 & -0.06 & 0.14 & 0.14 & $0.48 * * *$ \\
\hline 9 Sexual conflict & 0.01 & -0.10 & -0.01 & 0.06 & 0.10 & 0.03 \\
\hline I0 Attentional dysregulation & $-0.55^{* * *}$ & $-0.32 * * *$ & -0.12 & $0.17 * *$ & 0.13 & $0.19 * *$ \\
\hline II Peer rejection & $-0.22 * * *$ & $-0.72 * * *$ & $-0.32 * * *$ & 0.15 & 0.09 & 0.03 \\
\hline
\end{tabular}

I. Range of numbers reflects missing data for particular variables.

2. Criterion variables predicted to be most strongly associated (positively or negatively) with each factor are identified in bold.

SWAP-200-A, Shedler-Westen Assessment Procedure for Adolescents.

$* P<0.05, * * P<0.01, * * * P<0.001$.

personality traits and a distinct form of affectivity in which emotions spiral out of control. Emotional dysregulation appears to be associated with history of traumatic experiences such as sexual abuse and early parental separations (e.g. NakashEisikovits et al, 2003) and may be related to dysregulation of corticotrophin releasing factor, which has been linked both to childhood trauma and to later vulnerability to syndromes such as major depression and panic disorder (Heim \& Nemeroff, 2001).

Differences between the factor solution obtained here and the personality prototypes obtained from the same sample using Q-factor analysis are also instructive. It is noteworthy that the first six factors resemble six of the seven obtained $Q$ factors, which means that two very different ways of analysing the data - one identifying types (prototypes) and the other traits - yielded similar dimensions. However, the remaining scales identify traits or psychological functions that represent important aspects of personality pathology in adolescents but do not define a broader personality style: anxious obsessionality, delinquent behaviour, sexual conflict, attentional dysregulation and peer rejection.

\section{Potential objections and limitations}

This study has three primary limitations. The first is its exclusive reliance on a single informant (the treating clinician), which creates the possibility (like most studies of psychopathology, which rely exclusively on patient reports) of observer bias. Future research should attempt to replicate these findings using interview, informant and laboratory data as external criteria. Nevertheless, several factors limit this concern. First, SWAP-200 personality descriptions and ratings of adaptive functioning show high interrater reliability and validity and strongly predict relevant criterion variables as assessed by independent informants (Westen \& Muderrisoglu, 2003; Westen $\&$ Weinberger, 2004). Second, clinicians varied in their training (psychiatrists and psychologists) and theoretical orientations, and were unfamiliar with the factor structure of the instrument, minimising the likelihood of systematic sources of error stemming from rater biases. Finally, whereas factor analysis of the DSM-IV Axis II checklist in this sample produced a factor structure that strongly resembled the DSM-IV classification (Durrett \& Westen, 2005), factor analysis of the items of the SWAP-200-A, which include items assessing all of the Axis II criteria, did not. Thus, it is difficult to see how clinician biases could both lead to convergence with and divergence from the DSM-IV description of personality pathology in an adolescent sample.

A second potential objection is sample size. Clearly, the next step in this research requires a substantially larger sample, and such a study is now nearing completion (projected $n=1000$ ). Nevertheless, recent thinking about factor analysis, based on data from Monte Carlo simulations and other studies, suggests that factor solutions stabilise with far fewer cases than previously believed (often by 100 cases) as long as the factors are well marked by a sufficient number of items with loadings above 0.40 or 0.50 , as they were here (see Fabregar et al, 1999; Russell, 2002).

A final potential objection regards the question of the durability of personality pathology in adolescents and the appropriateness of diagnosing personality pathology at all in teenagers, an issue we have addressed elsewhere in detail (Westen \& Chang, 2000). The data presented here are cross-sectional, and future research should employ longitudinal designs. Nevertheless, recent research using different designs and measures suggests not only that personality can be assessed reliably in adolescents but that recognisable forms of personality pathology can be measured in adolescents and predict substantial variance in a range of outcomes, including outcomes measured longitudinally, above and beyond Axis I diagnosis (Ludolph et al, 1990; Johnson et al, 1999; Westen et al, 2003).

\section{Implications}

We note here two implications. First, research on adolescent psychopathology has often ignored personality variables because of the lack of appropriate constructs and 
measures. Availability of reliable and valid measures of adolescent personality pathology may prove useful in distinguishing clinical phenomena that may be quite distinct, such as different types of adolescents who share an Axis I mood disorder diagnosis.

Second, the data raise questions about whether we do better to characterise adolescent personality pathology in terms of the kinds of broad constellations connoted by the term 'personality disorder' or whether we might do better to focus on more specific traits that combine in various ways to form some of those constellations. Although we are far from a definitive answer to foundational questions such as these (with respect to either child and adolescent or adult psychopathology), one potential solution might be to combine both forms of classification. For example, an adolescent personality axis could include both a set of personality prototypes describing personality constellations as well as a small set of non-redundant traits such as attentional dysregulation or sexual conflict. From a clinical standpoint, aside from these descriptive (typological and trait approaches), another approach to diagnosis - explicit in the construction of the SWAP-200-A item set (which describes not only behaviours but also internal processes) - is functional assessment. A functional assessment focuses on understanding what is going right, what is going wrong and under what conditions certain pathological processes manifest for a given patient (i.e. the conditions under which certain functions go awry or break down). One way to reduce the gulf between clinicians and researchers is to decrease the gap between descriptive nosological constructs, which tend to be the focus of research, and functional constructs, which are essential to everyday clinical practice.

\section{ACKNOWLEDGEMENTS}

Preparation of this paper was supported in part by National Institute of Mental Health grants MH59685 and MH60892. The authors gratefully acknowledge the assistance of more than 300 clinicians who helped us refine the SWAP-200-A assessment instrument, including the 294 who participated in this study.

\section{REFERENCES}

Achenbach, T. M. (1991) Manual for the Child Behavior Checklist/4-18 and 1991 Profile. Burlington: University of Vermont, Department of Psychiatry.

\section{CLINICAL IMPLICATIONS}

- Personality pathology can be assessed in adolescents using the Shedler-Westen Assessment Procedure for Adolescents, which shows promise as a clinically and empirically useful instrument for assessing and taxonomising adolescent personality pathology.

- Negative affectivity (e.g. anxiety and depression) appears to be distinct from emotional dysregulation (the tendency for emotions to spiral out of control and motivate desperate measures to regulate it) as a personality trait, and the two constructs are likely to have different aetiologies and implications for treatment.

- A clinically useful, comprehensive personality axis for adolescents may need to incorporate both a set of personality prototypes or constellations similar to those described in DSM-IV, as well as a small set of traits such as delinquent behaviour, attentional dysregulation and sexual conflict, which do not describe personality styles but do represent enduring problems of adolescence.

\section{LIMITATIONS}

- Reliance on data from a single informant requires replication using criterion data from other sources.

Factor analysis with a large item set requires replication with a larger sample.

- Identification of personality constellations or traits in adolescents requires longitudinal research to study their course, change and development.

DREW WESTEN, PhD, Emory University, Atlanta, Georgia; LISSA DUTRA, EdM, Center for Anxiety and Related Disorders and Department of Psychology, Boston University, Boston, Massachusetts; JONATHAN SHEDLER, PhD, University of Denver, Denver, Colorado, USA

Correspondence: Dr Drew Westen, Department of Psychology and Department of Psychiatry and Behavioral Sciences, Emory University, 532 N. Kilgo Cir., Atlanta, Georgia 30322, USA. E-mail: dwesten@emory.edu

(First received 5 January 2004, final revision 9 September 2004, accepted 10 September 2004)

American Psychiatric Association (1994) Diagnostic and Statistical Manual of Mental Disorders (4th edn) (DSM-IV). Washington, DC: APA.

Cronbach, L. J. \& Meehl, P. E. (1955) Construct validity in psychological tests. Psychological Bulletin, 52 281-302

Durrett, C. \& Westen, D. (2005) The structure of axis II pathology in adolescents: A factor- and cluster-analytic investigation. Journal of Personality Disorders, in press.

Dutra, L., Campbell, L. \& Westen, D. (2004) Quantifying clinical judgment in the assessment of adolescent psychopathology: reliability, validity, and factor structure of the Child Behavior Checklist for Clinician-Report. Journal of Clinical Psychology, 60 65-85.

Fabregar, L. R., Wegener, D. T., MacCallum, R. C., et al (1999) Evaluating the use of exploratory factor analysis in psychological research. Psychological Methods, 4, 272-299.

Hare, R. D. (1998) Psychopaths and their nature: implications for the mental health and criminal justice systems. In Psychopathy: Antisocial, Criminal, and Violent Behavior (edsT. Millon \& E. Simonsen), pp. 188-212. New York: Guilford.

Heim, C. \& Nemeroff, C. B. (200I) The role of childhood trauma in the neurobiology of mood and anxiety disorders: preclinical and clinical studies. Biological Psychiatry, 49, 1023-1039.

Johnson, J. G., Cohen, P., Brown, J., et al (1999) Childhood maltreatment increases risk for personality disorders during early adulthood. Archives of General Psychiatry, 56, 600-606.

Levy, K. N., Becker, D. F., Grilo, C. M., et al (1999) Concurrent and predictive validity of the personality 
disorder diagnosis in adolescent patients. American Journal of Psychiatry, 156, I522-1528.

Linehan, M. M. (1993) Cognitive-Behavioral Treatment of Borderline Personality Disorder. New York: Guilford.

\section{Livesley, W. J., Jang, K. L. \& Vernon, P. A. (1998)} Phenotypic and genetic structure of traits delineating personality disorder. Archives of General Psychiatry, 55 $94 \mid-948$.

Ludolph, P., Westen, D., Misle, B., et al (1990) The borderline diagnosis in adolescents: symptoms and developmental history. American journal of Psychiatry 147, 470-476.

McCrae, R. \& Costa, P. L. (1997) Personality trait structure as a human universal. American Psychologist, 52, 509-516.

Nakash-Eisikovits, O., Dutra, L. \& Westen, D. (2003)

The relationship between attachment patterns and personality pathology in adolescents. Journal of the American Academy of Child and Adolescent Psychiatry, 4I IIII-II23.

Russell, D. W. (2002) In search of underlying dimensions: the use (and abuse) of factor analysis. Personality and Social Psychology Bulletin, 28, 1629-1646.
Shedler, J. \& Westen, D. (2004) Dimensions of personality pathology: an alternative to the Five Factor Model. American Journal of Psychiatry, 16I, 1743-1754.

Westen, D. (1991) Cognitive-behavioral interventions in the psychoanalytic psychotherapy of borderline personality disorders. Clinical Psychology Review, II, $211-230$.

Westen, D. (1998) Case formulation and personality diagnosis: two processes or one? In Making Diagnosis Meaningful (ed. J. Barron), pp. III-138. Washington, DC: American Psychological Association Press.

Westen, D. \& Chang, C. (2000) Personality pathology in adolescence: a review. Adolescent Psychiatry, 25 $61-100$

Westen, D. \& Muderrisoglu, S. (2003) Reliability and validity of personality disorder assessment using a systematic clinical interview: evaluating an alternative to structured interviews. Journal of Personality Disorders, I7, 350-368.

Westen, D. \& Shedler, J. (1999a) Revising and assessin Axis II, Part I: developing a clinically and empirically valid assessment method. American journal of Psychiatry, I56, 258-272.
Westen, D. \& Shedler, J. (1999b) Revising and assessing Axis II, Part 2: toward an empirically based and clinically useful classification of personality disorders. American Journal of Psychiatry, 156, 273-285.

Westen, D. \& Shedler, J. (2000) A prototype matching approach to diagnosing personality disorders toward DSM-V. Journal of Personality Disorders, 14, 109-126.

Westen, D. \& Weinberger, J. (2004) When clinical description becomes statistical prediction. American Psychologist, 59, 595-613.

Westen, D., Muderrisoglu, S., Shedler, J., et al (1997) Affect regulation and affective experience: individual differences, group differences, and measurement using a Q-sort procedure. Journal of Consulting and Clinical Psychology, 65, 429-439.

Westen, D., Shedler, J., Durrett, C., et al (2003) Personality diagnosis in adolescence: DSM-IV axis II diagnoses and an empirically derived alternative. American Journal of Psychiatry, 160, 952-966.

Widiger, T. A., Costa, P. T. \& McCrae, R. R. (2002) A proposal for Axis II: diagnosing personality disorders using the five-factor model. In Personality Disorders and the Five-Factor Model of Personality (2nd edn) (eds P. T. Costa \& T. A. Widiger), pp. 43I-456. Washington, DC: American Psychological Association Press. 